\title{
Perception and Experience of Biologic Therapy in Atopic Dermatitis: A Qualitative Focus Group Study of Physicians and Patients in Europe and Canada
}

\author{
Mahreen Ameen (D) - Stephan Meller (D) - Andreas Pinter (D) • \\ Neil H. Shear (D) · Angele Soria (D) · (the BADEL Study Group)
}

Received: September 13, 2021 / Accepted: October 5, 2021 / Published online: October 26, 2021

(c) The Author(s) 2021

\begin{abstract}
Introduction: The Biologics in Atopic Dermatitis: Experiences \& Learnings (BADEL) project aims to improve real-life understanding of how, where, and when biologics can play a role in the treatment of atopic dermatitis (AD) from the perspective of healthcare professionals (HCPs) and patients.

Methods: Individual experiences of 24 patients with moderate-to-severe $\mathrm{AD}$ and who had been treated with biologic therapy (dupilumab) for $\geq 3-6$ months, and 20 HCPs with a sub-specialty interest in $\mathrm{AD}$ were collected by means of focus
\end{abstract}

M. Ameen $(\square)$

Royal Free London NHS Foundation Trust, Belsize Park, London NW3 2QG, UK

e-mail:m.ameen@nhs.net

S. Meller

Department of Dermatology, Medical Faculty, Heinrich-Heine-University, Duesseldorf, Germany

A. Pinter

Department of Dermatology, University Hospital Frankfurt am Main, Theodor-Stern-Kai 7, 60590

Frankfurt am Main, Germany

N. H. Shear

Professor Emeritus, Department of Medicine, University of Toronto, Toronto, ON, Canada

A. Soria

Department of Dermatology and Allergology, Sorbonne University, Tenon Hospital, Assistance Publique Hôpitaux de Paris, 4 rue de la Chine, 75020 Paris, France groups held in Canada, Germany, France, Italy and the United Kingdom. Dupilumab was the only biologic therapy available at the time of the study. Results: Most patients had suffered from AD for many years, particularly from itch and psychosocial issues, with $\mathrm{AD}$ negatively impacting all aspects of their life. They had experienced a long treatment journey and seen many dermatologists, enduring treatment delays and failures. They had been prescribed various therapies without long-term success. Biologics provided symptom improvement, offering many patients a near-normal quality of life. Side effects, especially conjunctivitis, were the greatest drawback, and there were a few issues with incomplete or unreliable efficacy. HCPs agreed that biologic therapy for $\mathrm{AD}$ in the majority of patients demonstrated rapid onset, good efficacy and tolerability, and are a viable option in patients who had exhausted all other treatment options. However, those patients who failed to sufficiently respond or developed intolerable adverse effects, particularly ocular symptoms, require alternative therapeutic options.

Conclusion: Biologics can provide a near-normal quality of life for many patients with AD. Patients with $\mathrm{AD}$ who have failed conventional therapies should be offered all such novel therapies. Education and good patient-HCP communication will enable patients to manage their disease and treatment expectations. Patients and HCPs alike eagerly await alternative targeted therapies, which will offer greater choice 
and flexibility.Keywords: Atopic dermatitis; Biologic therapy; Qualitative study; Physicians; Patients; Experiences

\section{Key Summary Points}

The Biologics in Atopic Dermatitis: Experiences \& Learnings (BADEL) project aims to improve the real-life understanding of how, where and when biologics can play a role in the treatment of Atopic dermatitis (AD) from the perspective of healthcare professionals (HCPs) and patients

Individual experiences of patients with moderate-to severe $\mathrm{AD}$ and who had been treated with biologic therapy (dupilumab) for $\geq 3-6$ months, and HCPs with a subspecialty interest in $\mathrm{AD}$ were collected by means of focus groups held in participating countries

The study investigated the extent to which currently available biologics are addressing the needs of HCPs and patients with AD, and which needs remain unmet; the extent to which these biologics control AD; and the experiences of HCPs prescribing and patients treated with biologics for $\mathrm{AD}$

Patients suffered from AD for many years, had seen many dermatologists, and been prescribed a variety of therapies without long-term success, with negative impact on all aspects of life

Biologics can provide the degree of symptom improvement to give many patients a near normal quality of life and, therefore, patients with long-standing disease or a recent diagnosis should be offered the opportunity to benefit from all such novel therapies

Education and good patient-HCP communication will enable patients to manage their disease and treatment expectations, while patients and HCPs eagerly await alternative biologic treatment options to enable more choice and flexibility

\section{INTRODUCTION}

Atopic dermatitis (AD) is a chronic inflammatory skin disorder characterised by recurrent eczematous plaques and intense pruritus [1, 2]. $\mathrm{AD}$ commonly starts in childhood, affecting onefifth of children [2]; recent estimates suggest global adult prevalence rates of persistent or adult-onset disease are $2.1-4.9 \%$ [3]. AD is associated with an increased risk of other atopic and allergic conditions, and mental health disorders such as anxiety and depression $[1,2]$. AD pathophysiology is multifactorial, with genetic predisposition, epidermal barrier dysfunction and immune system dysregulation among the major components leading to barrier impairment, sensitisation and inflammation [4]. Patients with AD experience painful itching flares and sleep disturbances, and endure the psychosocial effects of embarrassment, reduced self-esteem, isolation and stigmatisation from having a visible disease $[1,2]$. The burden of disease has substantial impact on quality of life (QoL), daily activities, and on social, academic and occupational life of patients and their families [5-7].

Guidelines recommend initial therapy focused on skin hydration with emollients, avoidance strategies, flare management with topical antiinflammatories and ultraviolet (UV) therapy [8]. Topical corticosteroids and topical calcineurin inhibitors continue to be the most widely used therapies [8]. For refractory cases, there are systemic immunosuppressive therapies [9]. More recently, novel targeted therapies (e.g. biologics and Janus kinase inhibitors) have been approved or are in clinical development $[4,10,11]$.

The Biologics in Atopic Dermatitis: Experiences \& Learnings (BADEL) project aims to improve the real-life understanding of how, where and when biologics can play a role in the treatment of $\mathrm{AD}$ from the perspective of healthcare professionals (HCPs) and patients. This study, conducted in 2020, used focus group meetings to assess the following: the extent to which currently available biologics are addressing the needs of HCPs and patients with $\mathrm{AD}$, and which needs remain unmet; the extent to which these biologics control $\mathrm{AD}$; and the main experiences of prescribing HCPs and patients with AD treated with 
biologics. At the time of the focus group meetings, the fully human monoclonal antibody dupilumab was the only novel therapy approved for the treatment of moderate-to-severe AD. Dupilumab targets the interleukin- 4 receptor alpha subunit, thus down-regulating the type 2 inflammatory response [12]. Dupilumab has proven to be a safe drug in all ages, including adolescents from the age of 12 years and elderly patients. In May 2020, dupilumab was approved by the US Food and Drug Administration in children from 6 to 11 years with weight-based dosing [13-17]. The recent expansion of available targeted therapies has radically changed the treatment landscape since the focus group meetings were held.

\section{METHODS}

\section{Participants}

Patients were 18 years and older with a confirmed diagnosis of moderate-to-severe AD for at least 12 months. Patients had been using biologics for at least 3-6 months and had previously received other treatments for moderate-to-severe $\mathrm{AD}$, such as cyclosporine, methotrexate, UV therapy, mid-to-high potency topical corticosteroids and/or topical calcineurin inhibitors.

HCPs were dermatologists/clinical specialists in $\mathrm{AD}$ and one nurse with a special interest in AD. All HCP had been treating patients with AD for a minimum of 5 years. Dermatologists/clinical specialists actively prescribed biologics to five or more patients and had at least 6 months of experience of treating patients with $\mathrm{AD}$ with biologics.

\section{Study Methodology}

Two focus groups were held in each participating country, one for HCPs and one for patients. A total of 24 patients were recruited from the five participating countries; one patient-focus group comprised four patients (Canada) and four patient-focus groups comprised five patients (Germany, France, Italy and the UK). Twenty HCPs participated in the focus groups; each HCP-focus group was formed of four HCPs in each of the five countries.
Participants were identified and invited to participate in the focus groups by their HCPs or the local AD/dermatology patient association. Virtual group interviews were held in May through July 2020 for HCPs and in June through September 2020 for patients, with each lasting approximately 150 min. Participants were asked to describe their experiences of $\mathrm{AD}$ and biologic therapy, and their overall perception of treatment (Box 1). Participants had approximately $30 \mathrm{~min}$ to discuss each of the three sections: experiences with $\mathrm{AD}$ and the $\mathrm{AD}$ journey; experiences and learnings with biologics; and overall perception of biologic treatment (Box 2). Situation-directed prompt themes (e.g. why do you believe..., what has been the impact...) were used for greater understanding.

\section{Analysis}

The online virtual interviews were transcribed and translated; they were then reviewed and summarised by theme by two research analysts experienced in healthcare market research data analysis, who provided an unbiased and representative report. Representative verbatim quotations were selected to highlight the opinions expressed during the focus groups.

\section{Ethical Considerations}

The focus groups were a market research project conducted by a professional organisation, on behalf of a pharmaceutical company, in accordance with legal and ethical guidelines. This study was performed in accordance with the Helsinki Declaration of 1964 and its later amendments. Only anonymous and retrospective information about learnings and experiences with biologics in $\mathrm{AD}$ were collected and hence not considered relevant for ethical review. The study has not been retrospectively assessed by an ethics committee. Due to the anonymity, size and qualitative nature of the focus groups, the findings cannot be considered generalisable, nor transferable. All patients who participated in the focus groups did so voluntarily, were granted appropriate compensation and signed Informed Consent forms. They were free to opt out at any stage. 


\section{BOX 1. HEALTHCARE \\ PROFESSIONALS' AND PATIENTS' \\ PRE-SURVEY QUESTIONNAIRE}

\section{Healthcare professionals' pre-survey questionnaire}

1. How important is choice/availability of biologics to you, i.e. that you will ultimately have a selection to choose from when treating $\mathrm{AD}$ patients?

[Answer options: a) Not at all important, b) Somewhat important, c) Very important]

2. Out of 10 patients treated with biologics for $\mathrm{AD}$, in how many can you expect to see treatment success based on your own definition [Answer options: a) $0-3$ patients, b) 4-6 patients, c) 7-10 patients]

3. Out of 10 patients treated with biologics for $\mathrm{AD}$, in how many do you only see a partial response based on your own definition?

[Answer options: a) 0-3 patients, b) 4-6 patients, c) 7-10 patients]

4. Out of 10 patients treated with biologics for $\mathrm{AD}$, how many will be in combination therapy with topical treatments?

[Answer options: a) 0-3 patients, b) 4-6 patients, c) 7-10 patients]

5. Out of 10 patients, in how many do you combine biologics with systemics (e.g. cyclosporine and/or methotrexate)?

[Answer options: a) 0-3 patients, b) 4-6 patients, c) 7-10 patients]

6. Is it easy for you to prescribe biologic therapy for $\mathrm{AD}$ ?

[Answer options: a) Not easy at all, b) Somewhat easy, c) Very easy]

7. To what extent are biologics helping you achieve your patient treatment targets?

[Answer options: a) Not helpful at all b) Somewhat helpful, c) Very helpful]

8. Are you satisfied overall with current treatment options for your AD patients?

[Answer options: a) Not satisfied at all, b) Somewhat satisfied, c) Very satisfied]

Patients' pre-survey questionnaire

1. How is $\mathrm{AD}$ affecting your quality of life right now?

[Answer options: a) Responsible for poor quality of life, b) Somewhat affecting my quality of life, c) Hardly affecting my quality of life]

2. How was AD affecting your quality of life before you tried biologic therapy?

[Answer options: a) Responsible for poor quality of life, b) Somewhat affecting my quality of life, c) Hardly affecting my quality of life]

3 Based on your experience with other treatments for $\mathrm{AD}$, how much of a positive difference would you say biologics have made for you?

[Answer options: a) Not much difference, b) Moderate difference, c) Significant difference]

4 What has your experience been in obtaining access to biologic therapy?

[Answer options: a) Difficult, b) Moderately easy, c) Very easy]

5. To what extent do you feel the biologic you are using is relieving your AD symptoms?

[Answer options: a) Not at all, b) To some extent, c) Fully]

6. How would you rank the experience of taking/administering biologic therapy?

[Answer options: a) Difficult, b) Moderately easy, c) Very easy]

7. To what extent are you satisfied overall with the biologic treatment for your AD?

[Answer options: a) Not at all satisfied, b) Somewhat satisfied, c) Very satisfied]

8. To what extent did you ask for the treatment versus your doctor suggesting it?

[Answer options: a) I suggested it, b) We discussed it/collaborative decision, c) My doctor suggested it]

9. How are you currently treated for your AD?

[Answer options: a) Biologic only, b) Biologic in combination with one/

more topical or other products, c) No longer treated with biologic, only other types of treatments]

10. When diagnosed with $\mathrm{AD}$, to what extent were you provided with sufficient information on $\mathrm{AD}$ and treatment?

[Answer options: a) None/very little information, b) Sufficient information, c) Too much/information too overwhelming]

11. How long have you been treated with a biologic for your AD?

[Answer options: a) 0-3 months, b) 3-6 months, c) More than 6 months] 
BOX 2. HEALTHCARE PROFESSIONALS' AND PATIENTS' GROUP DISCUSSION TOPICS

\section{RESULTS}

The following outcomes are the qualitative responses obtained from engaging directly with focus group participants.

\section{HCP group discussions}

Section 1. HCP experience with AD and the AD journey

- Considering the latest three to four patients with moderate-to-severe $\mathrm{AD}$, detail your typical experiences with a patient with $\mathrm{AD}$

- What do you believe to be the greatest challenges in treating AD patients?

- What AD therapies have you had the most success with, and why?

Section 2. Experiences and learnings with biologic therapy

- What have your experiences been in treating AD patients with biologic therapy so far?

- What percentage of your AD patients are withdrawing totally from biologics? Why?

- Do you face any challenges in prescribing biologic therapy? Which?

- Do you face any challenges with patient acceptance of biologic therapy?

Section 3. Overall perception of biologic treatment

- Considering real-life, practical aspects in your daily practice/seeing patients, what do you consider to be the top three advantages of biologic therapy and why?

- What do you consider to be the top three challenges associated with biologic therapy and why?

- How satisfied are you with biologic therapy? What could be done to make your every-day easier?

Patient group discussions

Section 1. Your experience with AD

- When did your first symptoms of AD appear, how have they evolved over time?

- What has your treatment journey been like?

- What are your greatest challenges in having AD?

Section 2. Experiences and learnings with biologic therapy

- What has your experience with biological therapy for your AD been like?

- Have you faced any challenges in gaining access to biologic therapy and, if so, which?

- Do you see yourself continuing long term with biologic therapy and why/why not?

Section 3. Overall perception of biologic treatment

- Considering an average day in your life, what do you consider to be the top three advantages of biologic therapy and why?

- What do you consider to be the top three challenges associated with biologic therapy and why?

- What could be done to make your every-day life easier when it comes to treating your AD? 


\section{Participants}

Twenty-four patients ( 8 males, 16 females) with $\mathrm{AD}$ and 20 HCPs (14 males, 6 females) were interviewed.

\section{Experiences with $\mathrm{AD}$}

\section{Patients' Journey from First AD Symptoms}

Patients reported having AD at birth or developing the disease in early childhood, and most observed a change in the nature and worsening of their disease throughout their lives.
AD impacted on patients' mental, emotional and physical wellbeing. Patients reported challenges in almost all aspects of life, particularly difficulty in sleeping due to pain and itching, cracked bleeding skin that is aggravated by dressing and wearing clothes, and sweating on physical exertion. Patients described their problems in planning activities, which impinged on their social lives and relationships. They
"I was born with $A D$, and they diagnosed it with a milky crust.... When I
was little it was exudative, they were more isolated sores but over time it [plaques] went all over the body but [were] much drier." (IT, Patient)
"I have suffered from AD my entire life and it got progressively worse and worse as I got older." (CA, Patient)

Patients see many different doctors/specialists and try numerous therapies including homeopathic remedies, but treatments tend to lose efficacy over time and cause significant side effects. referred to the psychological impact of their disease, with feelings of loneliness, shame, embarrassment, depression and lack of confidence.

“....we all used cortisone.... There are unspeakable side effects [of

treatment]. Then you realise at some point that it [treatment] no longer

works." (IT, Patient) 


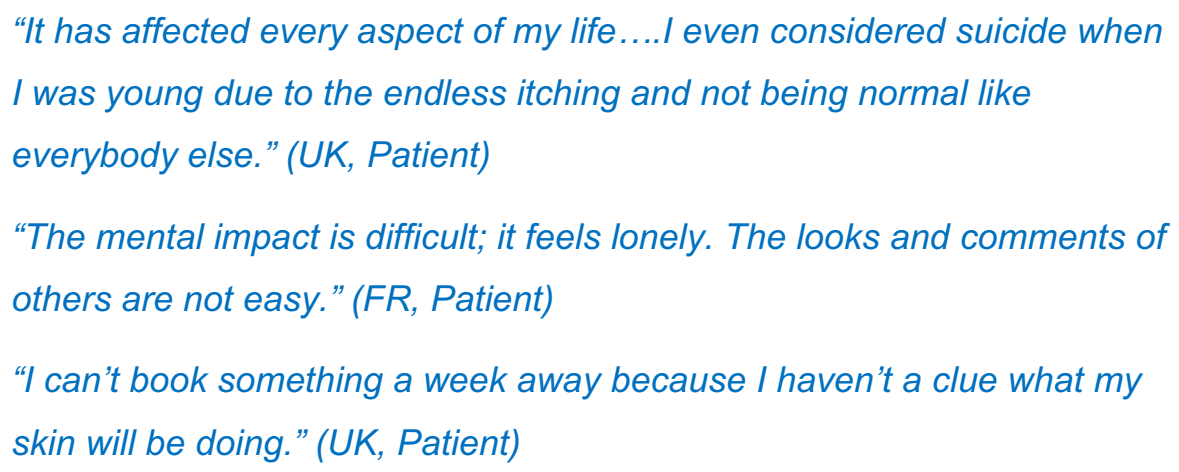

For some patients, a major issue was to find a dermatologist who could effectively relieve their disease and provide support. However, there is a fear of side effects, long-term damage and reduced efficacy. Despite years of doctor appointments, hospitalisations and treatment failure, patients remain hopeful that the next therapy will provide relief and so persist in trying new therapies.

"Then the roller coaster of always being hopeful.... always think this could be it, it could be a simple fix." (CA, Patient)

"Every couple of years I tried a new dermatologist, and then finally got one.... who didn't just dismiss me. He was like, 'We're going to follow up and see how this works and that works...." (CA, Patient)

HCPs' Experiences and Challenges of Treating $A D$

HCPs discussed and generally agreed that patients typically suffered with $\mathrm{AD}$ for years.

"Patients usually have an extensive history of disease, in terms of diagnostics and therapy, so they do end up with moderate-to-difficult courses." (DE, HCP) 
Their patients had seen many clinicians before coming under a specialist's care, at which time the disease symptoms have worsened.
Some HCPs believed that as new targeted therapies become available, they will be less hesitant to prescribe them and patients will be

"Most patients are referred....Many of them have seen multiple

physicians." (CA, HCP)

"They [patients] have such a high level of suffering due to the immense

itching and a loss of sleep." (DE, HCP)

HCP reported few treatment options available to either use or achieve efficacy long term for patients with AD. Partial or non-response to treatment and inconsistent results were common. Treatment was limited: topical steroids were a mainstay of treatment but there were efficacy and adherence issues. escalated to systemic therapy more rapidly. However, key challenges were educating

Topical steroids have been really great over the years and, if used well....can make all the difference. But there are a group of patients that.... will require something more." (UK, HCP)

In light of patients' long treatment journey, HCPs expressed the need for a safe and effective long-term therapy to resolve itching and appearance of lesions, stabilise the disease, eliminate flares and improve patients' QoL. patients about $\mathrm{AD}$ and its treatment, and overcoming their fear of systemic therapy.

"In my opinion, the main problem is the absence of medicines.

....Patients have been going on for years with only cortisone in most cases." (IT, HCP)

"....our aim as dermatologists is to try and manage the patient so their skin is as clear or nearly as clear as it can be." (UK, HCP) 


\section{"I have patients who are very nervous about systemic therapies." (FR, $\mathrm{HCP})$}

\section{Experiences with Biologic Therapy}

\section{Patients' Experiences}

Most patients considered that their QoL had improved with biologic therapy. They found the agent to rapidly provide at least reductions in symptoms of itch, cracked skin, sores and pain, with improvements in skin texture and sleep pattern.
Biologics were not effective for all patients and there were reports of less than complete efficacy with respect to skin condition, especially less than complete healing of the face.

\section{"I was able to feel so much better, live without pain, without sores, not scared to go out. I realised how I was living before. It's like everything that I was missing." (CA, Patient)}

However, patients treated with biologics also Flares and rebound symptoms (rash) were reported side effects and issues with adminisexperienced. tration (self-injecting), storage and cost. Side effects were most commonly eye related (especially conjunctivitis), but included various skinrelated effects and joint pain.

"I had as a complication blepharitis and initially treated it with

cyclosporine-based eye drops. But even with the side effects I think it's worth it." (IT Patient) 
"Unfortunately, dupilumab did not work for me." (IT, Patient)

"I do still have....little spots here and there...." (CA, Patient)

Patients assessed the drawbacks of side effects against the benefits of symptom relief and an improved QoL. The vast majority of patients believed that biologics were worth using despite the side effects, while one patient could not tolerate them for long periods. patients who had previously failed other systemic treatments responded favourably to biologics, providing pruritus relief and enabling sleep and improvement in QoL. HCPs reported that biologics are fast acting, with long-term efficacy, a good safety profile and reduce the need for topical treatment. However, a few
"I personally had a patient....little improved with respect to his skin, but
the patient absolutely did not want to stop the drug because he said that
the itching had passed." (IT, HCP)
"We've also had a lot of people with red faces....and one of them wanted
to stop [treatment]...." (UK, HCP)

\section{HCPs' Experiences}

HCPs' overall experience with biologics has been extremely positive. They found that some
HCPs mentioned that efficacy was limited for a minority of patients, where responses may be slow, partial or unstable, hampered by flares and eye-related side effects.

"We finally have something that works in the long term and that there are also relatively few side effects is at least a big step forward." (DE, HCP)

"Efficacy, safety, and quality of life are what I see, increasing very quickly." (IT, HCP)

"One of the things that I found that I didn't expect is that sometimes how slow it is to work and that, just by persevering for a little bit longer, sometimes you will avoid having to stop and change to another treatment." (UK HCP)

"I'd say a third of patients are partial responders to biologics." (CA, HCP) 
Some HCPs mentioned biologic discontinuation rates could be up to $5-10 \%$, predominantly due to tolerability issues and lack of efficacy. Notably, fear of COVID-19 infection was mentioned, owing to biologics causing an immunocompromised state. HCPs tended to establish a multidisciplinary approach to $\mathrm{AD}$ treatment with biologics, with ophthalmologists regularly being part of the team. frequency could manage side effects, while concomitant topical or systemic therapy could increase efficacy. The lack of alternative treatments at the time of the study made some HCPs
"There are a few patients, who, despite complete eye therapy and presentation to the ophthalmologists and the eye clinic, we did not manage to reduce this [conjunctivitis] side effect so that it was tolerable." $(D E, H C P)$
"I would say the primary failure rate is about $10 \%$ and the secondary failure rate about another 10 or $15 \%$ so I expect about a quarter of the patients on dupilumab to have trouble." (UK, HCP)

HCPs found that, where necessary, stopping and restarting treatment, and reducing dose reluctant to discontinue biologics or to initiate treatment in too many patients for fear of secondary failure that would leave no effective alternative treatment.

"We had some therapy failures.... With the combination therapies we naturally achieve even higher success rates...." (DE, HCP)

"Just because somebody hasn't responded right at that particular point in time, you can try again." (UK, HCP)

"When patients stop the biologic, we try to resume other treatments ....that's why we're looking forward to a variety of medications, ....so I can switch from one to the other depending on the side effects or the patient's tolerance or even the patient's preference." (FR, HCP) 


\section{Perceptions of Biologic Therapy}

\section{Patients' Perceptions}

Patients expressed their perceptions of biologics as feelings of normality, with less concern over the 'ordinary' things that people take for granted. They reported treatment advantages of enabling a longer sleep duration and comfortably wearing clothes without flaking skin on bedding and clothes, the pleasure of washing without pain, and participating in physical activities.

\section{HCPs' Perceptions}

HCPs reported the convenience of no baseline blood tests or frequent blood monitoring, and fewer patient visits. They reported satisfaction with patients' QoL improvement, mentioning convenient dosing frequency, stability of effect

“...I feel I've been given a chance to live a better life." (FR, Patient)

While dupilumab provided a simple dosing regimen, a few patients expressed concerns of treatment side effects and long-term impact, fear of treatment failure, cost and that the treatment was for life. and good side-effect profile. In a few cases, minor concerns were raised over patient response, side effects and costs, particularly

"The thought of being on something for the rest of your life, sometimes the thought of that poses a challenge." (CA, Patient)

with long-term use; however, those concerns never overshadowed the overwhelmingly positive feedback expressed in favour of biologics.

\footnotetext{
"So, for me it is the quality of life improvement with the stability, without large flare-ups, with the reliable tolerability, and that the patients really only have to see me routinely....every three months." (DE, HCP)

"The challenge is whether it will continue to be effective in the patient, to have few side effects over the long run." (FR, HCP)
} 


\section{Looking Ahead}

\section{Patients' Views}

Key themes consistently emerging as drivers of continued biologic use by patients were the advantages of a normal way of life outweighing the drawbacks of treatment; no other treatment provided a comparable level of efficacy and freedom from $\mathrm{AD}$; and that the treatment regimen fits well into many patients' lives, with some patients preferring bi-monthly subcutaneous injections to topical agents or tablets.
To make living with $\mathrm{AD}$ easier, patients wanted education for themselves, HCPs and the general public. They thought patient-focused education would help them better understand $\mathrm{AD}$ and its treatment, and cope with the psy-

"I don't want to suffer any more. So, I'm ready to continue this kind of treatment for the rest of my life." (FR, Patient)

Regardless of dupilumab's clear benefits, patients were open to new therapies that potentially offer improvements over dupilumab, such as: infrequent dosing by an alternative administration route (particularly oral); fewer side effects and improved tolerability; and greater/more consistent efficacy across body parts (especially face). chological effects of AD.

They suggested raising awareness of, and educating HCPs and the general public on, the impact of $\mathrm{AD}$ on daily life, to reduce the stigma

"I would not stop it. However, if there were any innovations where it

would do more....then I would abandon it." (IT, Patient)

of the disease. Further, they suggested easier access to biologic treatment, including broader availability and lower cost.

[With education] "You would have medical professionals who take it more

seriously and the general public not treat it like it's not a big deal." (CA,

Patient) 


\section{HCPs' Views}

While HCPs also reported favourably about dupilumab, they recognised the need for alternative treatments for patients who do not/partially respond to dupilumab, who experience side effects, or who have discontinued dupilumab. They were aware that patients who continued dupilumab despite a partial response, side effects or dislike of injections would readily switch to an alternative therapy. HCPs were attuned to new $\mathrm{AD}$ drugs in development, and keen to see how and where they would fit in the $\mathrm{AD}$ treatment armamentarium. improved to near 'normality' with biologic therapy because of reduced symptoms, better sleep and ability to carry out activities. However, biologics could be partially effective, have side effects, were costly and elicited fear about their long-term impact. Physicians expressed satisfaction in dosing frequency, reduced patient monitoring and visits, efficacy and side effect profile. However, concerns were raised regarding discontinuation rates because of tolerability issues and partial efficacy. Our findings

"As more and different options come to the market, we hope to have

other broader options to offer our patients as a biologic therapy." (CA,

$\mathrm{HCP})$

Conditions facilitating prescribing of biologic therapy for $\mathrm{AD}$ were cited as agents with more flexibility of dosing, fewer side effects, and patient education aimed at increasing acceptance of biologics. Many HCPs believe a program that provides financial support and access to 24-h patient support would enhance both HCPs' and patients' experience with biologic therapy for AD. add to the growing body of real-world literature on the AD experience and its management, and reflect discussions in a recent interview with a patient with $\mathrm{AD}$ and her consulting physician [18].

Patients with $\mathrm{AD}$ face a long treatment journey, starting early in life and causing con-

"Having flexibility in the dosing would be the thing that would make my

life much easier and the patients' living much easier." (UK, HCP)

\section{DISCUSSION}

This qualitative interview study provided personal accounts of experiences of patients living with $\mathrm{AD}$ and HCPs managing them. Patients considered that their QoL, in terms of their mental, emotional and physical wellbeing, had siderable psychosocial and physical burden. The significant negative impact of $\mathrm{AD}$ is consistent with other qualitative studies of AD [19-21]. Pruritus is responsible for much of the patients' disease burden [22], such as sleep disturbance [23], and lesions are associated with continual pain [24]. Thus, principal issues raised were the 
unrelenting itch-scratch cycle, pain, altered sleep patterns and symptom visibility.

The considerable effect of AD on psychosocial wellbeing is evidenced by its impact on interpersonal relationships, which leads to loneliness, depression, shame, poor self-image and stigmatisation $[19,20,25]$. Patients wish for better acceptance and understanding of $\mathrm{AD}$ in general [20]. AD limited patients' daily activities such as washing (painful) and physical activities (worsened itch) [19]. Some patients also report difficulties or missing days at work, which can lead to impaired performance and lack of funding to cover treatment costs, respectively $[19,20]$.

Being passed from one physician to another, receiving different diagnoses and trying various treatments is not uncommon for patients with $\mathrm{AD}$ [19]. Patients and carers have expressed dissatisfaction with the 'trial and error' approach to treatment [21, 26]. Patients have voiced frustration and helplessness with ineffective treatments, worry over poor $\mathrm{AD}$ understanding and awareness [19], and lack of new effective drugs to treat their disease [20].

In this study, the vast majority of patients using biologics experienced good relief from their AD symptoms for the first time in their lives, finding it to be 'life changing'. The most prevalent side effects were eye-related while other side effects may have been a consequence of previous treatments, such as bruising from long-term corticosteroid use and itching following their withdrawal. Evidence of long-term clinical efficacy and safety of dupilumab in patients with AD has been established in realworld clinical practice [27-29]. A real-world study found disease severity was improved in $86 \%$ of patients, $30 \%$ had complete skin clearance [28], and $29 \%$ of adverse events were ophthalmic [27]. We found that patients with $\mathrm{AD}$ receiving biologics made trade-offs between the treatments' benefits and living with any of a variety of side effects, continuing treatment because (at the time of the study) there was no equally effective alternative.

Key challenges in AD management included preventing flares and stabilising the disease, overcoming fear of systemic/ biologic therapies, patient education and an effective therapy that is safe for long-term use. Under-utilisation of systemic and biologics for inflammatory conditions is reported despite their efficacy and tolerability compared with corticosteroids [30-32]. A real-world study found that at 1 year, dupilumab persistence was $77 \%$, suggesting patients with $\mathrm{AD}$ were overall satisfied with treatment; $79 \%$ of those who discontinued dupilumab reinitiated therapy within 4 months [33]. Many issues are due to poor adherence to treatment [9, 34]. Risk perception among patients with inflammatory conditions appears to affect their fear of biologics, although they report willingness to accept risks for therapies offering significant therapeutic benefit [31]. Patient education interventions can help patients to better understand their disease, adhere to treatment and may assist in alleviating their concerns [9].

Effective treatment with a biologic was considered to be often delayed through lack of knowledge and expertise among GP/HCPs and that with education, patients may be escalated to biologic treatment sooner. This is recognised, with more patients now receiving the appropriate guidance from HCPs to benefit from these treatments [24].

We also found HCPs see patients less frequently when they are treated with biologics, because of the benefits of fewer blood level tests and less frequent monitoring. However, safety concerns over conjunctivitis mean that without adequate monitoring and care patients may prematurely discontinue therapy [24].

Biologics are considered to represent a breakthrough in $\mathrm{AD}$ management because of the improvement seen in clinical outcomes and quality of life of their patients [24]. However, while dupilumab (the only biologic available for $\mathrm{AD}$ at the time of this study) presents an advancement in $\mathrm{AD}$ therapy, there remains an unmet need for an effective agent for those patients for whom dupilumab is contraindicated or has low efficacy or adverse effects. AD management also needs to be generally improved because few patients with moderateto-severe $\mathrm{AD}$ receive the best treatment for their condition $[24,30]$.

A strength of our study is that we included patients and HCPs from several countries and 
therefore the perspectives are appropriate to different populations. Qualitative research enabled an understanding of the scope, indepth details and importance of the participant's experiences [35]. Prompting elicited directional and more detailed responses.

A limitation is the inclusion of relatively few participants who may not be representative of a population. Patient selection by HCPs may have meant inclusion of more severely affected patients, and we cannot be sure that the patients' contributions were not influenced by underlying comorbidities. Additionally, interviews were conducted in different languages, leading to possible translation or cultural bias.

\section{CONCLUSIONS}

This qualitative study collected the individual experiences of patients with AD and HCPs with an AD speciality. Many patients had suffered from $\mathrm{AD}$ for a considerable number of years, it negatively impacting on all aspects of life. Patients had seen many dermatologists and been prescribed a variety of therapies without long-term success. From our study, it is evident that biologics can provide the degree of symptom improvement to provide many patients with $\mathrm{AD}$ a near normal QoL. Patients who have long-standing disease or a recent diagnosis should be informed that there are effective treatments and be offered the opportunity to benefit from all available novel therapies. Education and good patient-HCP communication will enable patients to manage their disease and treatment expectations. Patients and HCPs alike eagerly await alternative biologic and other treatment options to enable more choice and flexibility.

\section{ACKNOWLEDGEMENTS}

The authors acknowledge the contributions of patients and physicians to the focus group discussions, making this research possible.

The BADEL Study group: Mahreen Ameen ${ }^{1}$, Stephan Meller ${ }^{2}$, Andreas Pinter ${ }^{3}$, Neil H Shear ${ }^{4}$, Angele Soria ${ }^{5}$. ${ }^{1}$ Royal Free London NHS
Foundation Trust, Belsize Park, London NW3 2QG, UK. ${ }^{2}$ Department of Dermatology, Medical Faculty, Heinrich-Heine-University, Duesseldorf, Germany. ${ }^{3}$ University Hospital Frankfurt am Main, Department of Dermatology, Theodor-Stern-Kai 7, 60590 Frankfurt am Main, Germany. ${ }^{4}$ Professor Emeritus, Department of Medicine, University of Toronto, ON, Canada. ${ }^{5}$ Sorbonne University, Department of Dermatology and Allergology, Tenon Hospital, Assistance Publique Hôpitaux de Paris, 4 rue de la Chine, 75020 Paris, France. Mahreen Ameen: ORCID iD: 0000-0002-7603-3007; Stephan Meller: ORCID iD: 0000-0002-3599-4346; Andreas Pinter: ORCID iD: 0000-0002-13301502; Neil H Shear: ORCID iD: 0000-0001-91511145; Angele Soria: ORCID iD: 0000-0002-87266658 .

Funding. This study was funded by LEO Pharma A/S, Ballerup, Denmark. LEO Pharma A/S, Ballerup, Denmark is also funding the journal's Rapid Service and Open Access Fees.

Authorship. Within the limits of this interview-based focus group study, all named authors meet the International Committee of Medical Journal Editors (ICMJE) criteria for authorship for this article, take responsibility for the integrity of the work as a whole, and have given their approval for this version to be published.

Author Contributions. All data generated as part of the qualitative focus group project is owned by LEO Pharma. LEO Pharma has neither had input nor influenced the contents or development of this manuscript, and the Authors have not been remunerated for their involvement. All authors, namely Mahreen Ameen, Stephan Meller, Andreas Pinter, Neil H Shear, and Angele Soria, had access to the aggregated data, provided substantial contribution and critical feedback of the drafts, and approved the final manuscript.

Medical Writing, Editorial, and Other Assistance. LEO Pharma (Denmark) has provided financial support for the manuscript writing assistance, which was provided by Sue 
Libretto, PhD, of Sue Libretto Publications Consultant Ltd (Hertfordshire, UK), and Copentown Healthcare Consultants (South Africa). LEO Pharma is the sponsor of the qualitative focus group project Biologics in Atopic Dermatitis: Experiences \& Learnings (BADEL) based on focus group sessions. Copentown organised and conducted the focus groups and interviews on behalf of LEO Pharma.

Prior Presentation. This is an original work and is not under consideration by any other journal. The data have not previously been presented or published in public.

Disclosures. Mahreen Ameen has been a principal investigator for clinical trials and/or scientific advisor and/or consultant for Abbvie, Eli Lilly, Pfizer, LEO Pharma, Regeneron, and Sanofi. Stephan Meller has received personal fees and/or grants from Amgen, Novartis, CSLBehring, LEO Pharma, AbbVie, Almirall, SanofiGenzyme, UCB Pharma, Lilly, Jansen Cilag, Milan, Medac, and Pfizer. Andreas Pinter has worked as an investigator and/or speaker and/or advisor for following pharmaceutical companies: AbbVie, Almirall-Hermal, Biogen Idec, Biontec, Boehringer-Ingelheim, Celgene, Celltrion, GSK, Eli-Lilly, Galderma, Hexal, Janssen, LEO Pharma, MC2, Merck Serono, Mitsubishi, MSD, Novartis, Pascoe, Pfizer, Tigercat Pharma, Regeneron, Roche, Sandoz Biopharmaceuticals, Sanofi-Genzyme, Schering-Plough, and UCB Pharma. Neil H Shear has been or is currently a consultant to AbbVie, Amgen, Bausch Medicine, Novartis, Sanofi-Genzyme, UCB, LEO Pharma, Ostuka, Janssen, Alpha Laboratories, Lilly, ChemoCentryx, Vivoryon, Galderma, Innovaderm, ChromoCell, Daichi, Kyowa Kirin, Alkyrmes, Argenx, Bristol Myers Squibb (BMS), Chemocentryx, KYE Pharma, Regeneron, and Reistone Pharma. Angele Soria has been and/or is a consultant and/or a speaker for Novartis, Sanofi-Genzyme, Abbvie, and Lilly.

Compliance with Ethics Guidelines. The focus groups were a market research project conducted by a professional organisation, on behalf of a pharmaceutical company, in accordance with legal and ethical guidelines. This study was performed in accordance with the Helsinki Declaration of 1964 and its later amendments. Only anonymous and retrospective information about learnings and experiences with biologics in AD were collected and hence not considered relevant for ethical review. The study has not been retrospectively assessed by an ethics committee. Due to the anonymity, size and qualitative nature of the focus groups, the findings cannot be considered generalisable, nor transferable. All patients who participated in the focus groups did so voluntarily, were granted appropriate compensation and signed Informed Consent forms. They were free to opt out at any stage.

Data Availability. The data collected and analysed during the current study are available from the corresponding author on reasonable request.

Open Access. This article is licensed under a Creative Commons Attribution-NonCommercial 4.0 International License, which permits any non-commercial use, sharing, adaptation, distribution and reproduction in any medium or format, as long as you give appropriate credit to the original author(s) and the source, provide a link to the Creative Commons licence, and indicate if changes were made. The images or other third party material in this article are included in the article's Creative Commons licence, unless indicated otherwise in a credit line to the material. If material is not included in the article's Creative Commons licence and your intended use is not permitted by statutory regulation or exceeds the permitted use, you will need to obtain permission directly from the copyright holder. To view a copy of this licence, visit http:// creativecommons.org/licenses/by-nc/4.0/.

\section{REFERENCES}

1. Avena-Woods C. Overview of atopic dermatitis. Am J Manag Care. 2017;23(8 Suppl):S115-23 (PMID: 28978208). 
2. Weidinger S, Novak N. Atopic dermatitis. Lancet. 2016;387(10023):1109-22. https://doi.org/10.1016/ S0140-6736(15)00149-X.

3. Barbarot S, Auziere S, Gadkari A, et al. Epidemiology of atopic dermatitis in adults: results from an international survey. Allergy. 2018;73(6):1284-93. https://doi.org/10.1111/all.13401.

4. Kim J, Kim BE, Leung DYM. Pathophysiology of atopic dermatitis: clinical implications. Allergy Asthma Proc. 2019;40(2):84-92. https://doi.org/10. 2500/aap.2019.40.4202.

5. Drucker AM, Wang AR, Li WQ, Sevetson E, Block JK, Qureshi AA. The burden of atopic dermatitis: summary of a report for the national eczema association. J Invest Dermatol. 2017;137(1):26-30. https:// doi.org/10.1016/j.jid.2016.07.012.

6. Yang EJ, Beck KM, Sekhon S, Bhutani T, Koo J. The impact of pediatric atopic dermatitis on families: a review. Pediatr Dermatol. 2019; 36(1):66-71. https://doi.org/10.1111/pde.13727.

7. Stingeni L, Belloni Fortina A, Baiardini I, et al. Atopic dermatitis and patient perspectives: insights of bullying at school and career discrimination at work. J Asthma Allergy. 2021;14:919-28. https:// doi.org/10.2147/JAA.S317009.

8. Wollenberg A, Barbarot S, Bieber T, et al.; European Dermatology Forum (EDF), the European Academy of Dermatology and Venereology (EADV), the European Academy of Allergy and Clinical Immunology (EAACI), the European Task Force on Atopic Dermatitis (ETFAD), European Federation of Allergy and Airways Diseases Patients' Associations (EFA), the European Society for Dermatology and Psychiatry (ESDaP), the European Society of Pediatric Dermatology (ESPD), Global Allergy and Asthma European Network (GA2LEN) and the European Union of Medical Specialists (UEMS). Consensus-based European guidelines for treatment of atopic eczema (atopic dermatitis) in adults and children: part I. J Eur Acad Dermatol Venereol. 2018;32(5):657-682. https://doi.org/10.1111/jdv. 14891.

9. Wollenberg A, Barbarot S, Bieber T, et al.; European Dermatology Forum (EDF), the European Academy of Dermatology and Venereology (EADV), the European Academy of Allergy and Clinical Immunology (EAACI), the European Task Force on Atopic Dermatitis (ETFAD), European Federation of Allergy and Airways Diseases Patients' Associations (EFA), the European Society for Dermatology and Psychiatry (ESDaP), the European Society of Pediatric Dermatology (ESPD), Global Allergy and Asthma European Network (GA2LEN) and the European Union of Medical Specialists (UEMS). Consensus-based European guidelines for treatment of atopic eczema (atopic dermatitis) in adults and children: part II. J Eur Acad Dermatol Venereol. 2018;32(6):850-878. https://doi.org/10.1111/jdv. 14888.

10. Kearns DG, Uppal SK, Chat VS, Wu JJ. Phase 2 and 3 trials of JAK inhibitors for atopic dermatitis: a review. Practical Dermatol. 2020:50-54.

11. Schaper-Gerhardt K, Rossbach K, Nikolouli E, Werfel T, Gutzmer R, Mommert S. The role of the histamine $\mathrm{H}_{4}$ receptor in atopic dermatitis and psoriasis. Br J Pharmacol. 2020;177(3):490-502. https://doi.org/10.1111/bph.14550.

12. Thibodeaux Q, Smith MP, Ly K, Beck K, Liao W, Bhutani T. A review of dupilumab in the treatment of atopic diseases. Hum Vaccin Immunother. 2019;15(9):2129-39. https://doi.org/10.1080/ 21645515.2019 .1582403$.

13. Hansel K, Patruno C, Antonelli E, et al. Dupilumab in adolescents with moderate to severe atopic dermatitis: a 32-week real-world experience during the COVID-19 pandemic. Clin Exp Dermatol. 2021. https://doi.org/10.1111/ced.14862.

14. Stingeni L, Hansel K, Antonelli E, et al. Atopic dermatitis in adolescents: effectiveness and safety of dupilumab in a 16-week real-life experience during the COVID-19 pandemic in Italy. Dematol Ther. 2021:e15035. https://doi.org/10.1111/dth.15035.

15. Patruno C, Napolitano M, Argenziano G, DADEDupilumab for Atopic Dermatitis of the Elderly study group, et al. Dupilumab therapy of atopic dermatitis of the elderly: a multicentre, real-life study. J Eur Acad Dermatol Venereol. 2021;35(4): 958-64. https://doi.org/10.1111/jdv.17094.

16. Patruno C, Fabbrocini G, Longo G, Dupilumab for Atopic Dermatitis of the Elderly (DADE) Study Group, et al. Effectiveness and safety of long-term dupilumab treatment in elderly patients with atopic dermatitis: a multicenter real-life observational study. Am J Clin Dermatol. 2021;22(4):581-6. https://doi.org/10.1007/s40257-021-00597-5.

17. Puar N, Chovatiya R, Paller AS. New treatments in atopic dermatitis. Ann Allergy Asthma Immunol. 2021;126(1):21-31. https://doi.org/10.1016/j.anai. 2020.08.016.

18. Berruyer M, Delaunay J. Atopic dermatitis: a patient and dermatologist's perspective. Dermatol Ther (Heidelb). 2021. https://doi.org/10.1007/s13555021-00497-w.

19. Marron SE, Cebrian-Rodriguez J, Alcalde-Herrero VM, Garcia-Latasa de Aranibar FJ, Tomas-Aragones L. Psychosocial Impact of Atopic Dermatitis in Adults: A Qualitative Study. Actas Dermosifiliogr. 
2020;111(6):513-517. https://doi.org/10.1016/j.ad. 2019.03.018.

20. Ring J, Zink A, Arents BWM, et al. Atopic eczema: burden of disease and individual suffering - results from a large EU study in adults. J Eur Acad Dermatol Venereol. 2019;33(7):1331-40. https://doi.org/10. $1111 /$ jdv.15634.

21. Santer M, Burgess H, Yardley L, et al. Experiences of carers managing childhood eczema and their views on its treatment: a qualitative study. Br J Gen Pract. 2012;62(597):e261-7. https://doi.org/10.3399/ bjgp12X636083.

22. Fleming $\mathrm{P}$, Yang YB, Lynde $\mathrm{C}, \mathrm{O}^{\prime}$ Neill B, Lee KO. Diagnosis and management of atopic dermatitis for primary care providers. J Am Board Fam Med. 2020;33(4):626-35. https://doi.org/10.3122/jabfm. 2020.04.190449.

23. Jeon C, Yan D, Nakamura M, et al. Frequency and management of sleep disturbance in adults with atopic dermatitis: a systematic review. Dermatol Ther (Heidelb). 2017;7(3):349-64. https://doi.org/ 10.1007/s13555-017-0192-3.

24. Fougerousse AC. At the dawn of a therapeutic revolution for atopic dermatitis: an interview with Dr Anne-Claire Fougerousse. Dermatol Ther (Heidelb). 2021. https://doi.org/10.1007/s13555-021-00489W.

25. Brunner PM, Silverberg JI, Guttman-Yassky E, Councilors of the International Eczema Council, et al. Increasing comorbidities suggest that atopic dermatitis is a systemic disorder. J Invest Dermatol. 2017;137(1):18-25. https://doi.org/10.1016/j.jid. 2016.08.022.

26. Howells LM, Chalmers JR, Cowdell F, Ratib S, Santer M, Thomas KS. "When it goes back to my normal I suppose": a qualitative study using online focus groups to explore perceptions of "control" among people with eczema and parents of children with eczema in the UK. BMJ Open. 2017;7(11):e017731. https://doi.org/10.1136/bmjopen-2017-017731.

27. Sears AV, Woolf RT, Gribaleva E, et al. Real-world effectiveness and tolerability of dupilumab in adult atopic dermatitis: a single-centre, prospective 1-year observational cohort study of the first 100 patients treated. Br J Dermatol. 2020. https://doi. org/10.1111/bjd.19631.

28. Wang C, Kraus CN, Patel KG, Ganesan AK, Grando SA. Real-world experience of dupilumab treatment for atopic dermatitis in adults: a retrospective analysis of patients' records. Int $\mathrm{J}$ Dermatol. 2020;59(2):253-6. https://doi.org/10.1111/ijd. 14573 .

29. Kim NS, Maliyar K, Oliveira L, O'Toole A, Gooderham MJ. Real-world experience of dupilumab in the treatment of moderate-to-severe atopic dermatitis. Int J Dermatol. 2020;59(10):e361-3. https://doi. org/10.1111/ijd.15053.

30. Pascal C, Maucort-Boulch D, Gilibert S, et al. Therapeutic management of adults with atopic dermatitis: comparison with psoriasis and chronic urticaria. J Eur Acad Dermatol Venereol. 2020;34(10):2339-45. https://doi.org/10.1111/jdv. 16329.

31. De Felice KM. Patients perception of risks and benefits of biologic therapy. Inflamm Bowel Dis. 2020;26(1):147-9. https://doi.org/10.1093/ibd/ izz122.

32. Fougerousse AC, Jacobzone C, Mery-Bossard L, GEM ResoEczema Group, et al. Use of systemic medications for treating adult atopic dermatitis in france: results of a practice survey. Clin Cosmet Investig Dermatol. 2021;14:179-83. https://doi.org/ 10.2147/CCID.S300402.

33. Silverberg JI, Guttman-Yassky E, Gadkari A, Kuznik $A$, et al. Real-world persistence with dupilumab among adults with atopic dermatitis. Ann Allergy Asthma Immunol. 2021;126(1):40-5. https://doi. org/10.1016/j.anai.2020.07.026.

34. Feldman SR, Cox LS, Strowd LC, et al. The challenge of managing atopic dermatitis in the United States. Am Health Drug Benefits. 2019;12(2):83-93.

35. Keeley T, Williamson P, Callery $\mathrm{P}$, et al. The use of qualitative methods to inform Delphi surveys in core outcome set development. Trials. 2016;17(1): 230. https://doi.org/10.1186/s13063-016-1356-7. 